\title{
Home-Based Exercise Training During COVID-19 Pandemic in Post-Bariatric Patients: a Randomized Controlled Trial
}

\author{
Gersiel Nascimento de Oliveira Júnior ${ }^{1}$ - Karla Fabiana Goessler ${ }^{1}$ • Jhonnatan Vasconcelos Pereira Santos ${ }^{1}$. \\ Alisson Padilha de Lima ${ }^{1}$ • Rafael Genário ${ }^{1}$. Carlos Alberto Abujabra Merege-Filho ${ }^{1}$. Diego Augusto Nunes Rezende ${ }^{1}$.

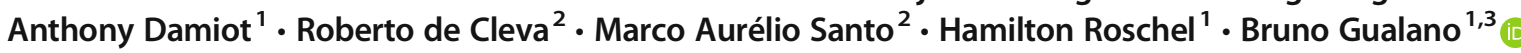

Received: 29 March 2021 / Revised: 13 July 2021 / Accepted: 20 July 2021 / Published online: 7 August 2021

(C) The Author(s), under exclusive licence to Springer Science+Business Media, LLC, part of Springer Nature 2021

\begin{abstract}
This data is mandatory Please provide.
\end{abstract}

Keywords COVID-19 $\cdot$ Home-based exercise $\cdot$ Bariatric surgery

The adoption of a healthy lifestyle is deemed important to sustain bariatric surgery-induced health benefits [1]. During COVID-19 pandemic, a set of social distancing measures, including stay-at-home orders, have increased physical inactivity among patients who had bariatric surgery. In addition, several of them have been refrained from face to face health care. Consequently, these patients are shown to be at risk for overall health deterioration [2]. Home-based exercise programs have been postulated as a potential tool to maintain health status during pandemic [3]. The aim of this randomized

Gersiel Nascimento de Oliveira Júnior and Karla Fabiana Goessler contributed equally to this work.

\section{Bullet Points}

- Out of concern that COVID-19 pandemic has created a situation where at-risk patients are confined with limited access to face-to-face health care, we performed a randomized clinical trial to investigate the health impact of a home-based exercise training program in post-bariatric patients.

- The intervention was overall ineffective, except for slight, but significant improvements in selected functional outcomes. The attrition rate was high and the compliance to training was unexpectedly low, suggesting that patients may have found it difficult to adhere to the program.

- These data have the potential to inform new home-based interventions, which may possibly incorporate more practical/feasible forms of exercise delivery and behavioral strategies focused on better engaging patients after bariatric surgery.

Bruno Gualano gualano@usp.br

1 Applied Physiology and Nutrition Research Group, School of Physical Education and Sport, Faculdade de Medicina FMUSP, Universidade de São Paulo, São Paulo, SP, Brazil controlled trial was to investigate the impact of a home-based exercise training program in patients who had surgery and were provisionally deprived from in-hospital health care. Data were collected between April and December 2020 in Sao Paulo (Brazil), a period in which social distance measures were in place. This study was approved by the local ethical committee, and all patients provided written informed consent. The trial was registered at ClinicalTrials.gov (NCT04425005).
2 Department of Digestive Surgery, School of Medicine, University of Sao Paulo, São Paulo, SP, Brazil

3 Rheumatology Division, Hospital das Clinicas HCFMUSP, Faculdade de Medicina, Universidade de São Paulo, São Paulo, SP, Brazil 
Men and women who had Roux-en-Y gastric bypass $(n=50)$ and sleeve gastrectomy $(n=17)$ within the past 12 months and had not been presently involved in exercise training were eligible. Exclusion criteria were post-operatory period lower than 3 months or exceeding 12 months, physical limitations precluding exercise, or diagnosed with mental disorders or COVID-19 upon recruitment.

The home-based exercise training group (HB) underwent a 3-month, 3-times-a-week, semi-supervised training program (one weekly session was supervised by a trained researcher through live videoconference calls; and the remaining two weekly sessions were monitored through text messaging). Sessions consisted of a 5-min light warm-up followed by seven strengthening exercises and aerobic exercise (walking and/or running) through remote guidance (supplementary file 1). Overall, $38 \%$ of sessions were monitored by videoconference and $62 \%$ were followed by text messages. For those who completed the study (attrition rates are described below), training attendance was $79.6 \%$ and $51.5 \%$ met the prescribed intensity. The control group (CRTL) received no intervention (Figs. 1, 2, 3, 4, 5, 6 and 7).

Clinical parameters, blood sample, and health-related questionnaires were assessed at baseline and after 3 months. Data were collected either at patient's home by our staff or at our intrahospital laboratory, depending on the availability of the patient to attend the laboratory. Waist circumference was the primary outcome.
Office blood pressure (BP) was measured three times after a 10-min seated rest, using an automated device (Omron HEM-7320®). Blood sample was collected after a 12-h fasting to assess glucose, insulin, glycated hemoglobin (HbA1c), C-peptide, lipid profile, and C-reactive protein (CRP). Functional capacity was evaluated by handgrip-strength test and 30-s sit-to-stand test, whereas aerobic fitness was assessed by step test to predict $\mathrm{VO}_{2}$. Height, weight, and hip circumference were also assessed. Patients completed a demographic questionnaire including information about age, sex, self-reported use of medication, and comorbidities. Patients were inquired about socioeconomic status [4], depression and anxiety symptoms [5, 6], health-related quality of life [7], and physical activity [8] by phone calls.

Data were primarily analyzed using intentionto-treat-principles (ITT). Dependent variables were tested by generalized estimation equations (GEE). Bonferroni post-hoc test was performed for multiple comparisons. In a complete case approach, between-group delta changes were compared using independent $t$ tests. Categorical variables were analyzed by Fisher exact test. Significance level was set at $p \leq 0.05$ (SPSS, version 20.0).

Seventy-six patients were randomized. Among them, $5(13.5 \%)$ and $7(21.2 \%)$ in CTRL and HB, respectively, were only remotely assessed as they lived outside the city. Four patients from HB withdrew their consents before baseline data collection [severe depressive symptoms $(n=1)$, not available for training program $(n=2)$,
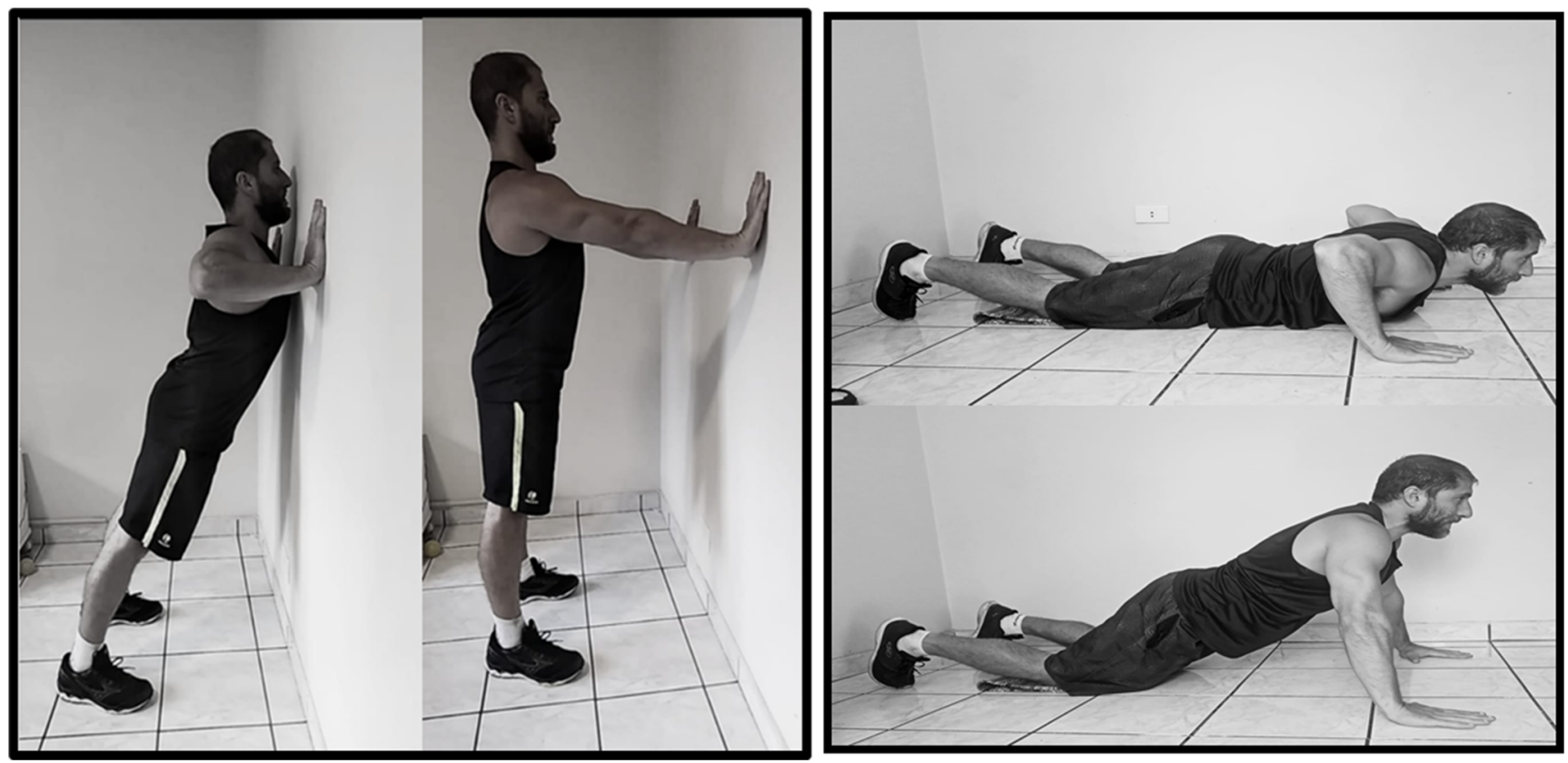

Fig. 1 Wall or floor push-up 


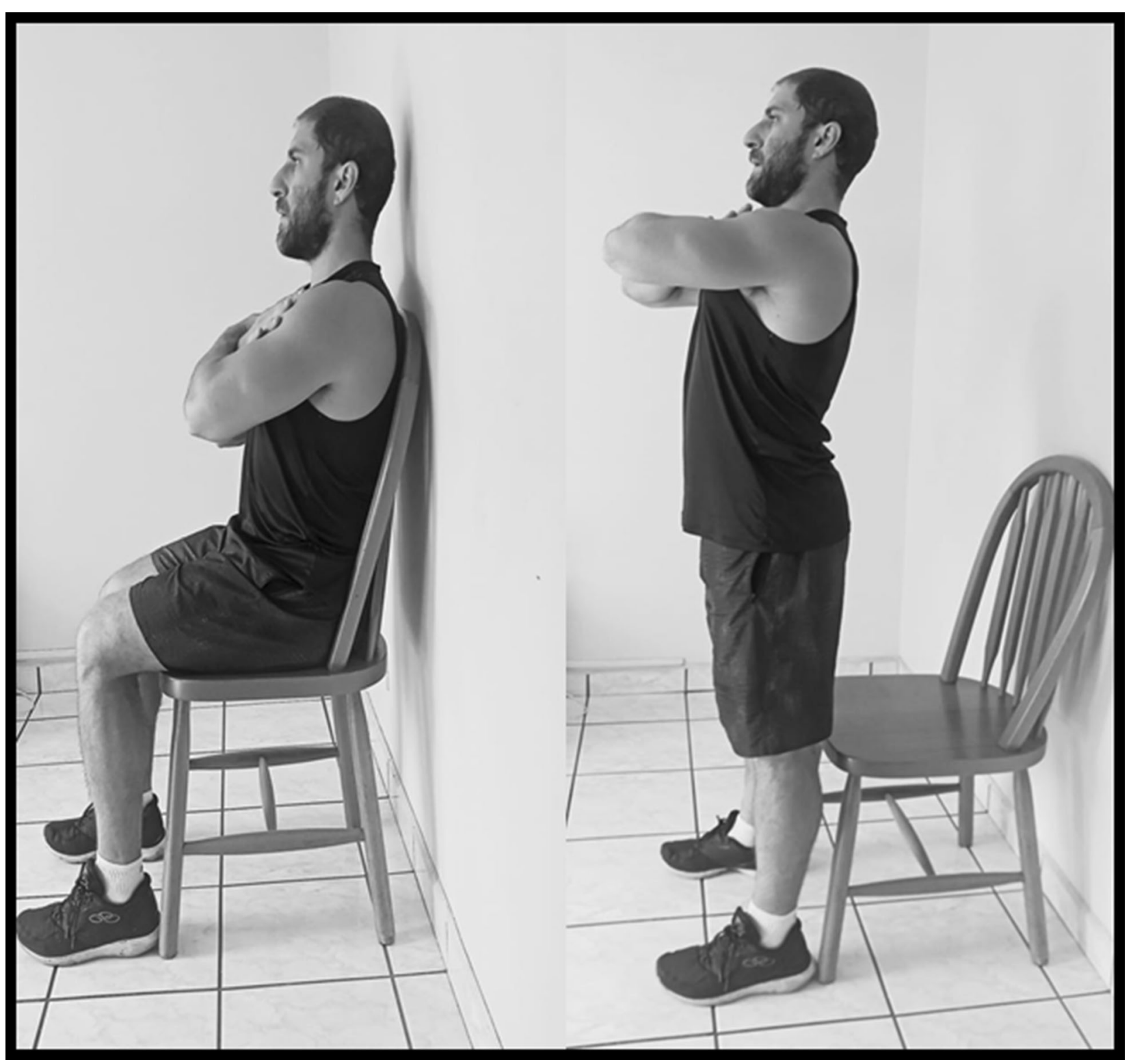

Fig. 2 Sit-to-Stand

Fig. 3 Unilateral rowing

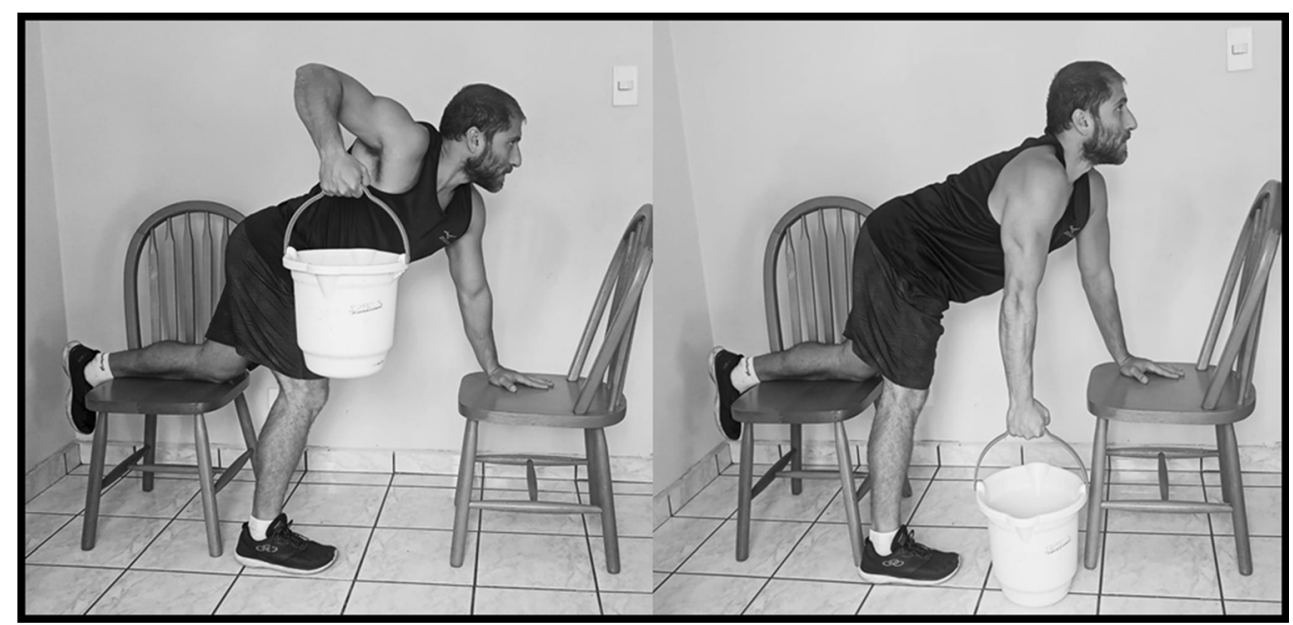




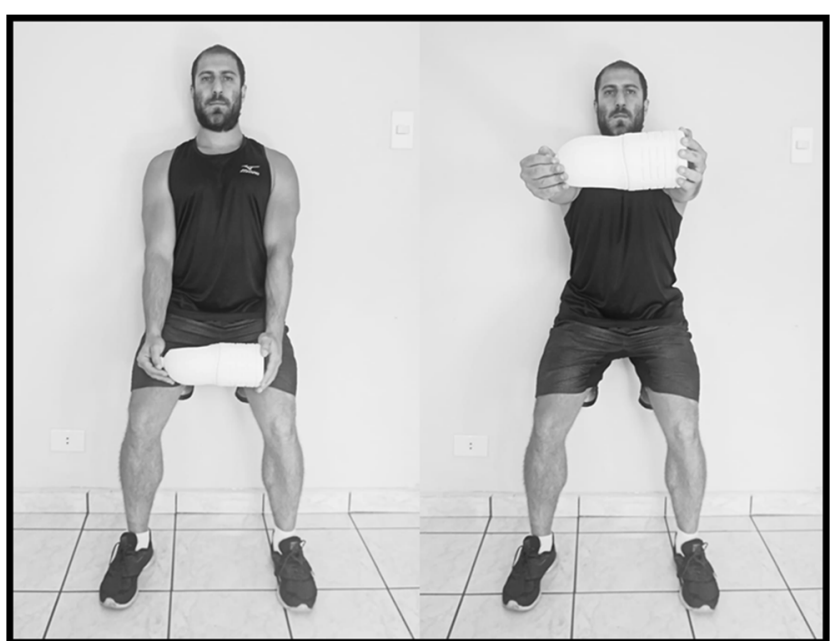

Fig. 4 Front raise

and not available for assessments $(n=1)]$ and were excluded from the trial. Two additional patients (CTRL: $n=1$ and HB: $n=1$ ) were also excluded after randomization due to a delay between randomization and baseline data collection, exceeding the pre-specified post-surgery period limit (i.e.; $<12$ months). None of these patients underwent baseline data collection and were excluded from the ITT analysis. Nineteen patients were lost to follow up (CTRL: $n=$ 5 and HB = 14) but were included in ITT analysis (Figure S1). Patients' main characteristics are shown in Table 1.

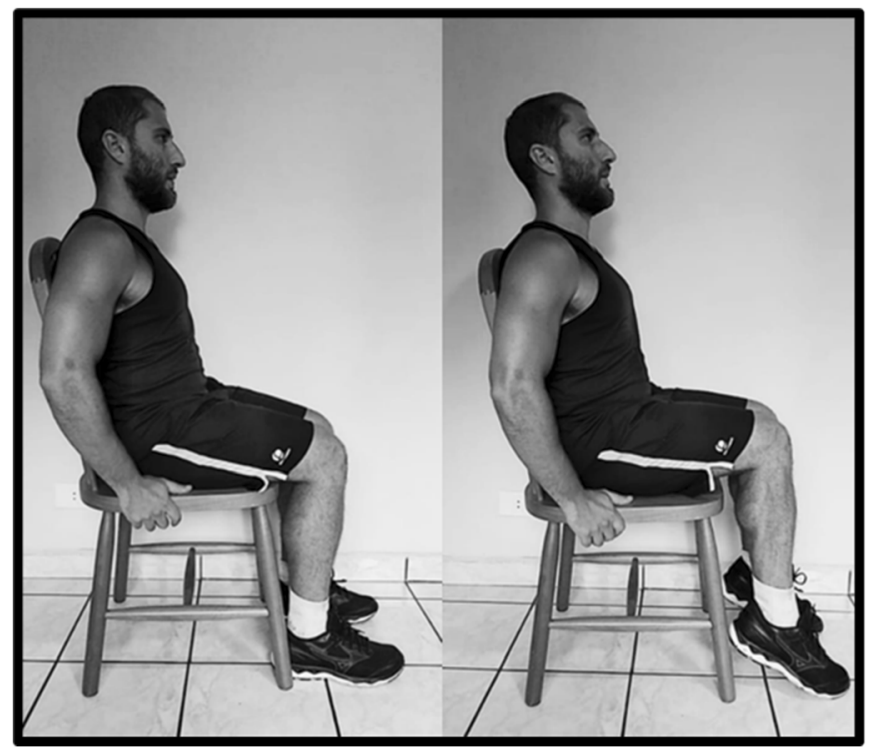

Fig. 5 Seated or stand calf

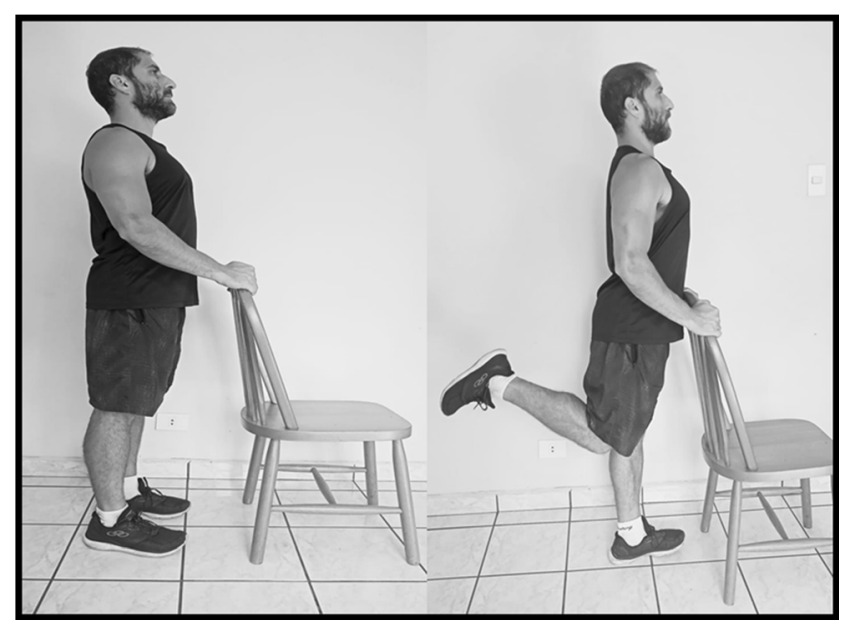

Fig. 6 Leg curl

Waist circumference did not change within or between groups $(p>0.05)$. Main effects of time were found for hip circumference, weight, BMI, HDL, CRP, and diastolic BP, without any group by time interactions (Table 2). Global physical and mental health, anxiety and depression symptoms, and physical activity did not change throughout the study (all $p>0.05$, data not shown).

Both ITT and case-complete analyses showed that HB vs. CTRL improved 30-s sit-to-stand test performance $(p<0.01$ and $p=0.02)$ and predicted $\mathrm{VO}_{2}(p$ $=0.04$ and $p=0.04$ ) (Table 2).

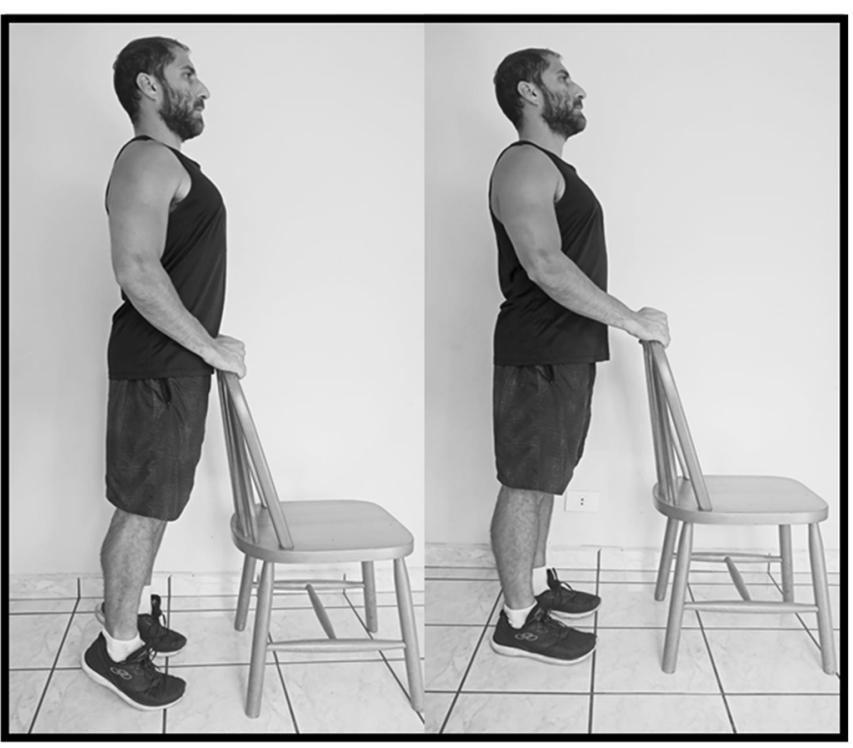




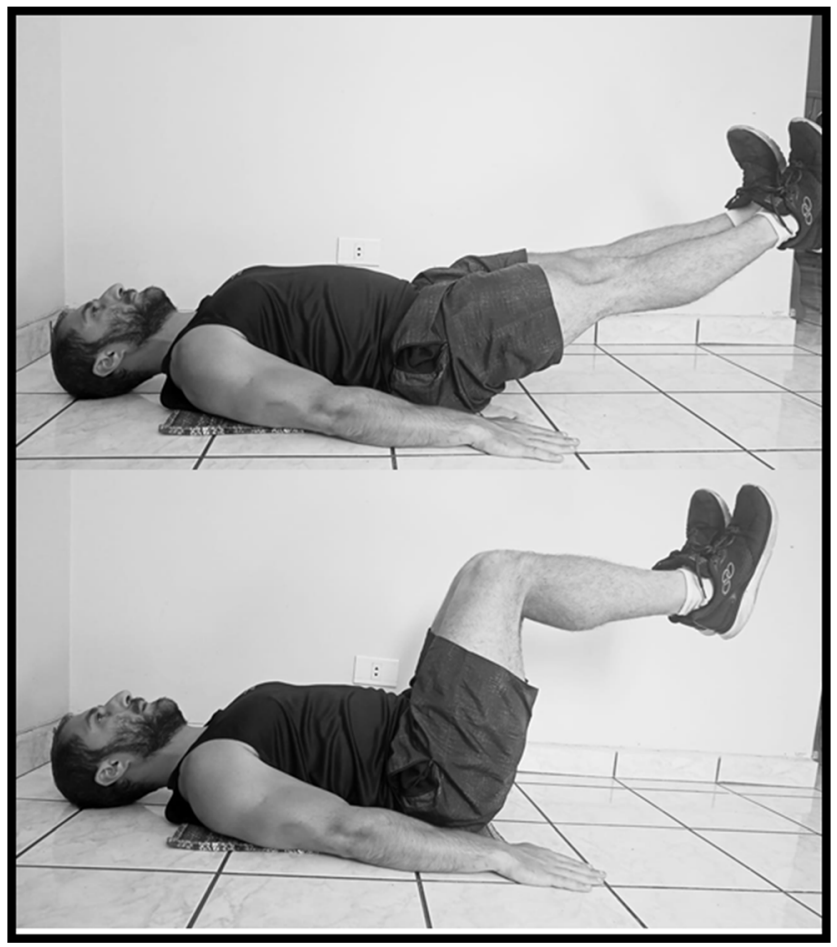

Fig. 7 Abdominal
Home-based exercise has emerged as an alternative tool to delivery physical activity to self-isolated individuals due to COVID-19 outbreak. However, the viability and efficacy of this intervention remain underexplored. Recently, we showed that patients who had bariatric surgery have experienced cardiometabolic disturbances, poor quality of life, and inactivity during pandemic [2]. This trial tested whether such a poor condition could be lessened by a home-based exercise program.

The intervention was overall ineffective, except for slight, but significant improvements in selected functional outcomes. The limitations of this study may have contributed for the null findings. The main limitation was the small sample size and the significant number of patients lost at follow-up, especially from HB group. In addition, training compliance was unexpectedly low, suggesting that patients may have found it difficult to adhere to the program. These data have the potential to inform new home-based interventions, which may possibly incorporate more practical/feasible forms of exercise delivery and behavioral strategies focused on better engaging patients who have bariatric surgery.
Table 1 Baseline characteristics according to allocation group

\begin{tabular}{lll}
\hline Baseline characteristic & CTRL & HB \\
\hline Sex $(\boldsymbol{n}, \boldsymbol{\%})$ & & $28(84.8)$ \\
Female & $31(83.8)$ & $5(15.2)$ \\
Male & $6(16.2)$ & $47.5 \pm 11.6$ \\
Age (years), mean \pm DP & $47.3 \pm 10.9$ & $8(3-12)$ \\
Post-surgery period (months), median (min-max) & $7.0(3-12)$ & \\
Type of surgery $(\boldsymbol{n}, \boldsymbol{\%})$ & & $21(67.7)$ \\
Gastrointestinal bypass Y-in-Roux & $29(80.6)$ & $10(32.3)$ \\
Sleeve gastrectomy & $7(19.4)$ & $17(53.1)$ \\
Physical activity $(\boldsymbol{n}, \boldsymbol{\%})$ & & $8(25.0)$ \\
Active & $14(38.9)$ & $7(21.9)$ \\
Irregularly active & $9(25.0)$ & $2(6.1)$ \\
Inactive & $13(36.1)$ & $31(93.9)$ \\
Smoking habits $(\boldsymbol{n}, \boldsymbol{\%})$ & & $7(30.4)$ \\
Yes & $4(10.8)$ & $4(15.4)$ \\
No & $33(89.2)$ & $3(11.5)$ \\
Medication $(\boldsymbol{n}, \boldsymbol{\%})$ & & $0(0.0)$ \\
Antihypertensive & $16(50.0)$ & \\
Antidiabetic & $7(21.9)$ & \\
Antihypothyroid & $3(9.4)$ & $1(3.1)$ \\
Antidepressant & & \\
Socioeconomic status $(\boldsymbol{n}, \boldsymbol{\%})$ & $0(0.0)$ & $30(100.0)$ \\
C & & \\
D-E & & \\
\hline & & \\
\hline
\end{tabular}

CTRL control group, $H B$ home-based exercise training group 


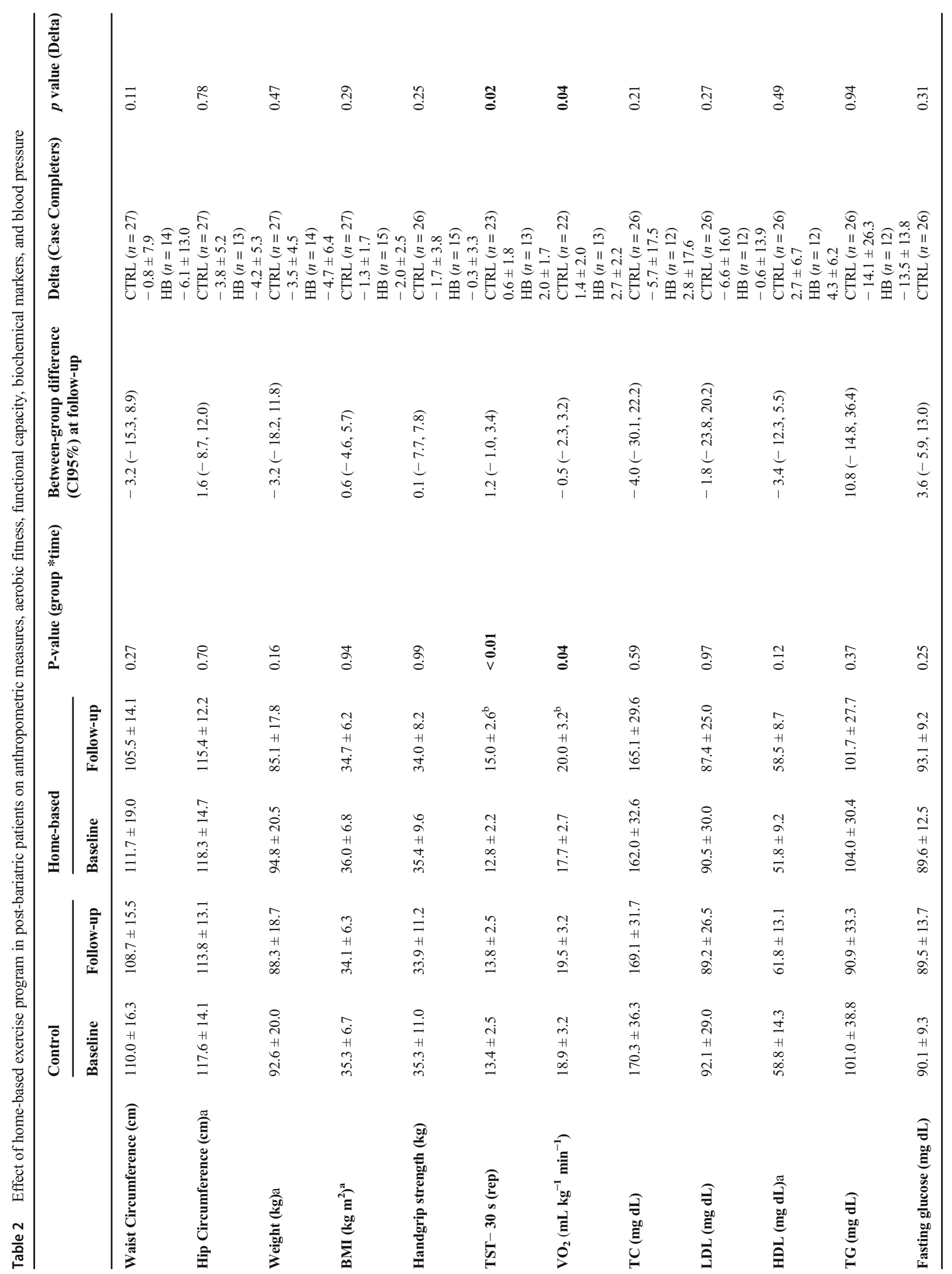




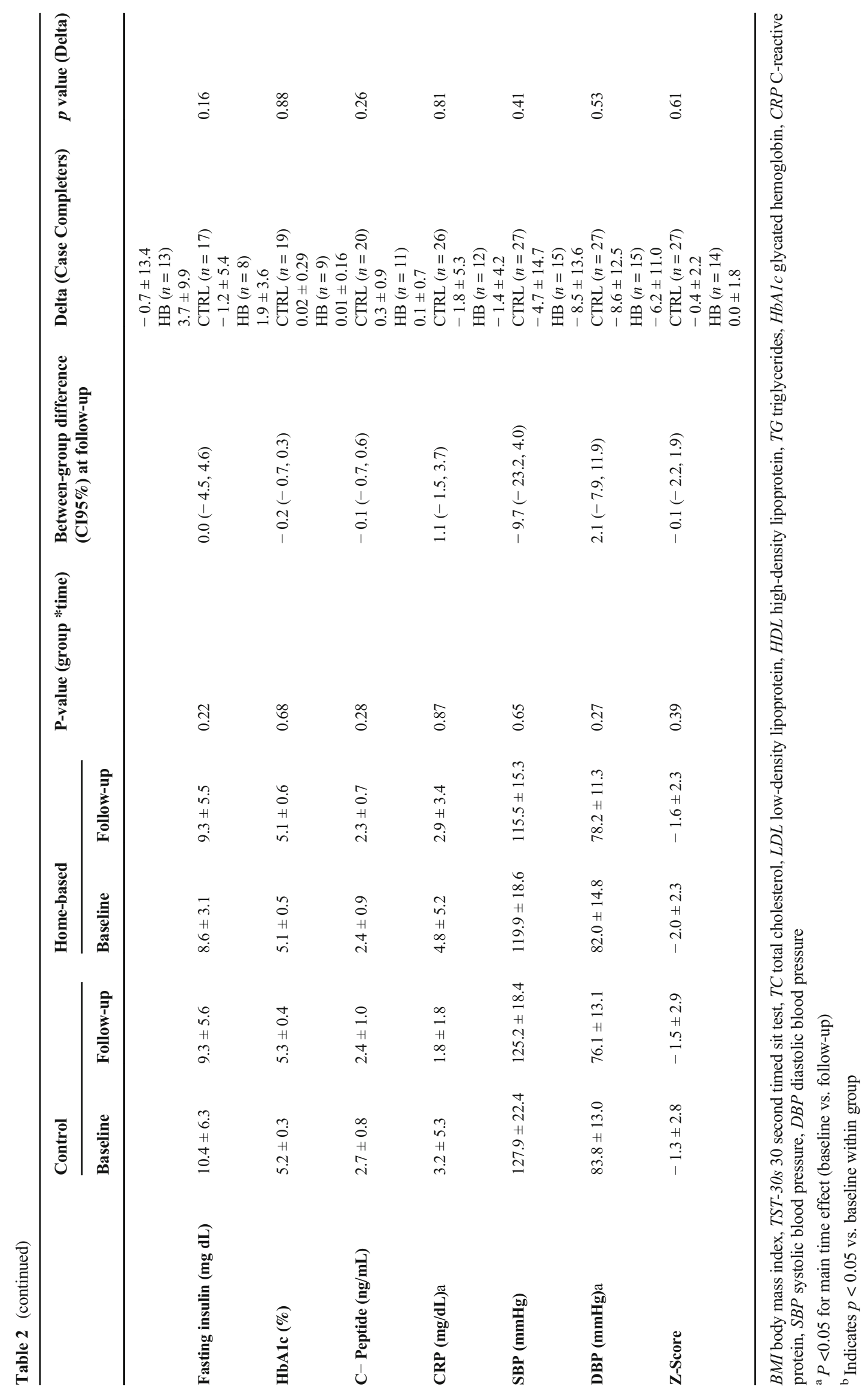


Supplementary Information The online version contains supplementary material available at https://doi.org/10.1007/s11695-021-05621-5.

Funding Sao Paulo Research Foundation - FAPESP (grants \#2017/ 16104-0, \#2017/13552-2, \#2019/18039-7, \#2019/22160-6) and CAPES (88887.473556/2020-00).

\section{Declarations}

Ethics Approval and Consent to Participate All procedures performed in studies involving human participants were in accordance with the ethical standards of the institutional and/or national research committee and with the 1964 Helsinki declaration and its later amendments or comparable ethical standards.

Conflict of Interest The authors declare no conflict of interest

\section{References}

1. Dantas WS, Gil S, Murai IH, Costa-Hong V, Pecanha T, MeregeFilho CAA, et al. Reversal of improved endothelial function after bariatric surgery is mitigated by exercise training. J Am Coll Cardiol. 2018;72(18):2278-9.

2. Goessler KF, Nicoletti CF, Rezende DAN, Sieczkowska SM, Esteves GP, Genario R, Oliveira Júnior GN, Meireles K, Pinto AJ, Nakahara-Melo M, Cleva R, Santo MA, Kirwan JP, Roschel H,
Gualano B. Outpatient screening of health status among postbariatric patients during the COVID-19 pandemic in Sao Paulo, Brazil. Obesity (Silver Spring). 2020;28(12):2263-4.

3. Pecanha T, Goessler KF, Roschel H, Gualano B. Social isolation during the COVID-19 pandemic can increase physical inactivity and the global burden of cardiovascular disease. Am J Physiol Heart Circ Physiol. 2020;318(6):H1441-H6.

4. ABEP. Critério de Classificação Econômica Brasil. 2015; Available from: www.abep.org.

5. Beck AT, Epstein N, Brown G, Steer RA. An inventory for measuring clinical anxiety: psychometric properties. J Consult Clin Psychol. 1988;56(6):893-7.

6. Beck AT, Ward CH, Mendelson M, Mock J, Erbaugh J. An inventory for measuring depression. Arch Gen Psychiatry. 1961;4:56171.

7. Ware Jr JE, Sherbourne CD. The MOS 36-item short-form health survey (SF-36). I. Conceptual framework and item selection. Med Care. 1992;30(6):473-83.

8. Matsudo S, Araújo T, Matsudo V, Andrade D, Andrade E, Oliveira LC, et al. International physical activity questionnaire (IPAQ): Study of validity and reliability in Brazil. Revista Brasileira de Atividade Física e Saúde. 2001;6(2).

9. Thompson PD, Arena R, Riebe D, Pescatello LS. American College of Sports M. ACSM's new preparticipation health screening recommendations from ACSM's guidelines for exercise testing and prescription, ninth edition. Curr Sports Med Rep. 2013;12(4):215-7.

Publisher's Note Springer Nature remains neutral with regard to jurisdictional claims in published maps and institutional affiliations. 